\title{
The carboxy-terminal tail of the homeo domain protein $\alpha 2$ is required for function with a second homeo domain protein
}

\author{
Arkady Mak and Alexander D. Johnson ${ }^{1}$ \\ Department of Biochemistry and Biophysics, ${ }^{1}$ Department of Microbiology and Immunology, University of California, San \\ Francisco, San Francisco, California 94143-0502 USA
}

\begin{abstract}
The homeo domain protein $\alpha 2$ from Saccharomyces cerevisiae has two roles in the a/ $\alpha$ cell: With MCM1, $\alpha 2$ turns off transcription of a-specific genes; with a1 (a second homeo domain protein), $\alpha 2$ represses transcription of haploid-specific genes. From the carboxy-terminal side of the $\alpha 2$ homeo domain extends an unstructured 22-amino-acid residue tail. In this paper we show that the carboxy-terminal tail of $\alpha 2$ is required for formation of a stable a1/ $\alpha 2-0$ perator complex and is thus required for a1/ $\alpha 2$-mediated repression of transcription. In contrast, the tail is dispensable for $\alpha 2 / M C M 1-m e d i a t e d$ repression. These results indicate that a short, unstructured tail mediates the interaction between two homeo domain proteins.
\end{abstract}

[Key Words: $\alpha 2$; al; homeo domain proteins; protein-DNA interaction; protein-protein interaction]

Received June 4, 1993; revised version accepted August 4, 1993.

The products of the mating type $(M A T)$ locus maintain cell type in yeast by controlling the expression of sets of cell type-specific genes (for reviews, see Herskowitz 1989; Sprague 1990; Dolan and Fields 1991; Johnson 1992). In particular, a cells, haploid cells that carry the $M A T$ a allele, express a-specific genes. In contrast $\alpha$ cells, haploid cells that carry $M A T \alpha 1$ and $M A T \alpha 2$ (together called $M A T \alpha$ ), do not express a-specific genes. In these cells the MAT $\alpha 2$ product $(\alpha 2)$, in conjunction with the protein MCM1, recognizes the a-specific gene operator, located upstream of each a-specific gene, and represses transcription of these genes. In the same cells, the $M A T \alpha 1$ product $(\alpha 1)$ turns on expression of $\alpha$-specific genes. In a/ $\alpha$ diploid cells, which result from the mating of an a cell with an $\alpha$ cell, $\alpha 2$ and MCM1 repress the a-specific genes as described above. In these cells $\alpha 2$ also works in conjunction with a1, the MATa gene product, to repress haploid-specific genes by binding with al to a distinct DNA sequence (the haploid-specific gene operator) found upstream of each haploid-specific gene. Because $M A T \alpha 1$ is itself repressed by the combination of $\alpha 2$ and $a 1$, the $\alpha$-specific genes remain unexpressed in a/ $\alpha$ cells. Thus $\alpha 2$ has two roles in the a/ $\alpha$ cell: With MCM1, it turns off transcription of a-specific genes; with a1, $\alpha 2$ represses transcription of haploid-specific genes.

The $\alpha 2$ protein is composed of two structural domains (see Fig. 1). The amino-terminal domain of $\alpha 2$ mediates $\alpha 2 / \alpha 2$ dimerization and is required for the repression function of $\alpha 2$; DNA binding is carried out by the homeo domain, which is located in the carboxy-terminal region. (Hall and Johnson 1987; Sauer et al. 1988; Wolberger et al. 1991; Komachi and Johnson, unpubl.). Between the amino-terminal domain and the homeo domain, the protein has a flexible hinge region that is used to contact MCM1 (Vershon and Johnson 1993). In addition to these regions, $\alpha 2$ has a short ( 22 amino acid) tail extending from the homeo domain to the carboxyl terminus of the protein. In this paper we show that the tail of $\alpha 2$ is required for al/ $\alpha 2$ repression but is dispensable for $\alpha 2 /$ MCM1 repression. We show further that the tail acts to stabilize the protein-DNA complex formed by $\alpha 2$, al, and the haploid-specific gene operator.

\section{Results}

The carboxy-terminal tail of $\alpha 2$ is protease sensitive

In general, structurally compact domains of a polypeptide are more resistant to proteolysis than are extended regions. The experiment shown in Figure 2 demonstrates that the carboxy-terminal tail of $\alpha 2$ is protease sensitive compared with the adjacent homeo domain. In this experiment $\alpha 2_{128-210}$, a fragment that consists of the homeo domain and the carboxy-terminal tail, was exposed to V8 endopeptidase. After the indicated amounts of time, the digestion products were visualized on a Coomassie-stained SDS-polyacrylamide gel following electrophoresis. As seen in Figure 2, $\alpha 2_{128-210}$ was converted, after short periods of digestion, to a slightly 


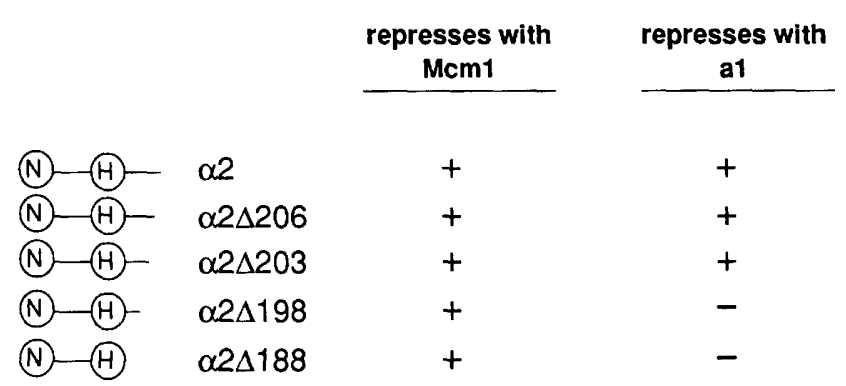

Figure 1. Behavior in vivo of carboxy-terminal truncations of $\alpha 2$. On the upper left is a schematic representation of $\alpha 2$ showing the amino-terminal domain $(\mathrm{N})$, the hinge, the homeo domain $(\mathrm{H})$, and the carboxy-terminal tail. The truncations shown below are designated by the number of the final residue. For each derivative, the ability to repress the a-specific genes (with MCM1) and the haploid-specific genes (with al) was assayed by mating tests and by expression of the appropriate reporter construct (for details, see Figs, 3A and 4).

smaller species that was relatively resistant to further proteolysis. Amino-terminal sequencing of the products of $40 \mathrm{~min}$ of digestion, at which time virtually all of the starting material had been converted to the smaller form, indicated that digestion was occurring at the carboxyl terminus. Thus, the carboxy-terminal end of $\alpha 2_{128-210}$ was sensitive to V8 protease, whereas the adjacent homeo domain was relatively resistant. Under the digestion conditions utilized in this experiment (see Materials and methods), V8 protease cleaves on the carboxy-terminal side of glutamate residues. $\alpha 2_{128-210}$ contains seven potential cleavage sites, two in the tail and five in the homeo domain. The results of the proteolysis indicate that the $\alpha 2$ tail has an extended structure distinct from that of the homeo domain. This view is consistent with the three-dimensional structure of $\alpha 2_{128-210}$ in solution and bound to DNA: The homeo domain is ordered and compact, but the carboxy-terminal tail is disordered (Phillips et al. 1991; Wolberger et al. 1991).

\section{Truncation of the $\alpha 2$ tail inactivates a1/ $\alpha 2$ repression but not a2/MCM1 repression in vivo}

We then determined whether the 22-amino-acid-residue tail of $\alpha 2$ has a function. As a first step, we made a series of carboxy-terminal truncations of $\alpha 2$ and examined the ability of the truncated proteins to supply $\alpha 2$ activity in vivo. As summarized in Figure 1, truncations that end with residues $206(\alpha 2 \Delta 206)$ or $203(\alpha 2 \Delta 203)$ of $\alpha 2(210$ residues full length) resemble the wild-type $\alpha 2$ in their behavior in vivo (Mak 1992). However, $\alpha 2 \Delta 188$, which lacks the entire carboxy-terminal tail, and $\alpha 2 \Delta 198$, which retains only the first 10 amino acids after the homeo domain, fail to function with $\mathbf{a} 1$ in directing repression of the haploid-specific genes. These $\alpha 2$ derivatives are still capable of repressing the a-specific genes with MCM1, demonstrating that these truncations inactivate only a subset of the $\alpha 2$ functions. What follows is a detailed characterization of the most extensive of the mu- tant truncations, $\alpha 2 \Delta 188$. We consider first the behavior of the $\alpha 2 \Delta 188$ truncation with respect to $\alpha 2 / \mathrm{MCM} 1$ mediated repression of a-specific genes.

Two physiological tests show that $\alpha 2 \Delta 188$ is capable of repressing a-specific genes. Several a-specific genes, such as MFa1 and MFa2, which encode the structural genes for a-factor (Brake et al. 1985), and STE6, whose gene product is required for the secretion of a-factor (Kuchler et al. 1989), are involved in the production and secretion of a-factor, a pheromone secreted by a cells. a-Factor secretion can be detected as a halo of growth arrest surrounding a patch of cells placed on a lawn of $\alpha$ cells. The halo is produced because a-factor arrests the growth of the $\alpha$ cells that comprise the lawn. A strain deleted for the $M A T$ locus (mat $\Delta$ ) produces an a-factor halo (Fig. 3A, line 1) because the a-specific genes are expressed. When the $\alpha 2 \Delta 188$ allele is introduced into this strain on a plasmid, the halo is no longer made, indicating that $\alpha 2 \Delta 188$ turns off at least one gene required for a-factor production and secretion.

In this experiment, the plasmid that provided $\alpha 2 \Delta 188$ also carried a wild-type $M A T \alpha 1$ gene. As shown in Figure $3 \mathrm{~A}$, mat $\Delta$ cells carrying this plasmid produced $\alpha$-factor, a pheromone normally produced by $\alpha$ cells. As described in the introductory section, $\alpha 1$ turns on transcription of the $\alpha$-specific genes, including the structural genes for $\alpha$-factor $[M F \alpha 1$ and $M F \alpha 2$ (Kurjan and Herskowitz 1982; Singh et al. 1983)]. However, formation of an $\alpha$-factor halo also requires that an a-specific gene, BAR1 /which encodes a protease that degrades $\alpha$-factor; see MacKay et al. 1988), be turned off. Because the plasmid-containing strain produces $\alpha$-factor, we can conclude that $\alpha 2 \Delta 188$ also turns off the $B A R 1$ gene. These results indicate that $\alpha 2 \Delta 188$ efficiently turns off at least two, and probably all (see below), of the a-specific genes.

The same type of physiological test was also utilized to examine the ability of $\alpha 2 \Delta 188$, in combination with al, to repress the haploid-specific genes. Normally, a/ $\alpha$

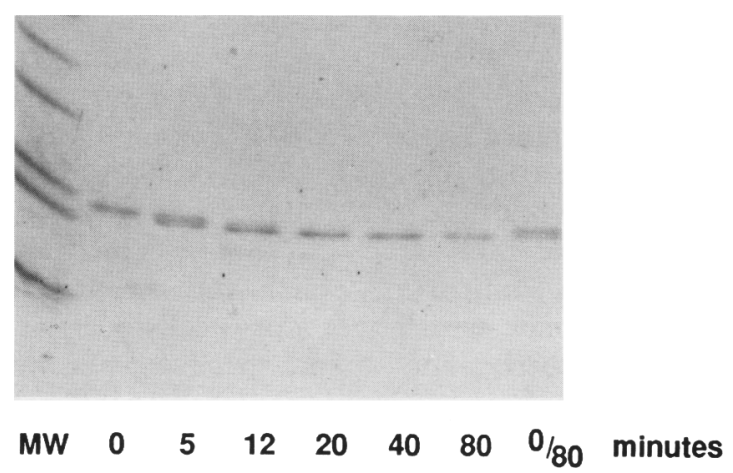

Figure 2. The carboxy-terminal tail of $\alpha 2$ is protease sensitive. A fragment of $\alpha 2\left(\alpha 2_{128-210}\right)$ that consists of the homeo domain and the carboxy-terminal tail was exposed to V8 endoproteinase for varying lengths of time, as indicated along the bottom. Following digestion, the reaction products were displayed on a Coomassie-stained SDS-polyacrylamide gel. The lane marked $0 / 80$ is a $1: 1$ mixture of the products of 0 and $80 \mathrm{~min}$ of proteolysis; the leftmost lane displays molecular weight markers. 
A

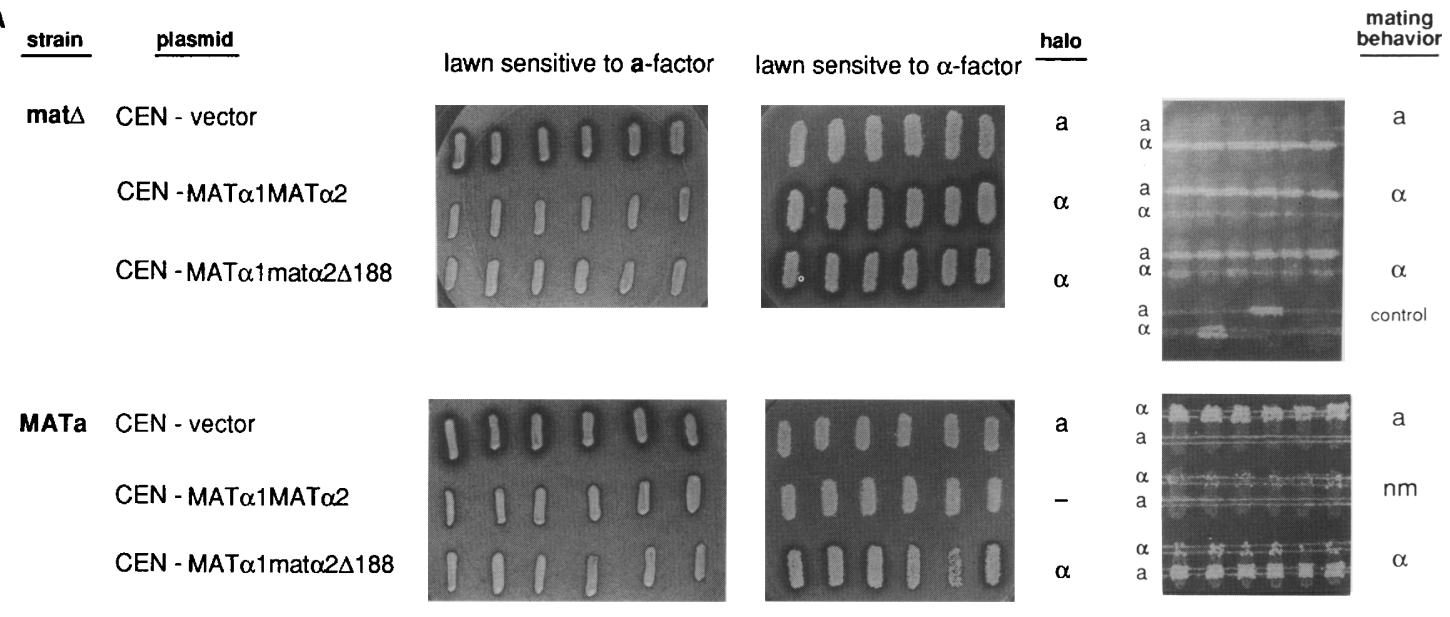

Figure 3. mat $2 \Delta 188$ brings about repression of a-specific genes but not haploid-specific genes. $(A)$ a-Factor production, $\alpha$-factor production, and mating ability were assayed in both mat $\triangle$ and $M A T$ a cells carrying the plasmids indicated. Six independent transformants of each plasmid were patched out on lawns of cells sensitive to a-factor (left) or to $\alpha$-factor (right). Patches producing a halo when grown on a lawn sensitive to a-factor (left) are said to make an a halo; those producing a halo on a lawn sensitive to $\alpha$-factor (right) make an $\alpha$ halo. For mating tests, the transformants were patched and cross-stamped with $\alpha \mid M A T \alpha$ lys1) and a (MATa cry1 lys1) strains (as labeled to the left of each row of mating

$\mathrm{B}$

\begin{tabular}{ccc} 
strain & plasmid & sporulation \\
\cline { 3 - 3 } mat $\Delta$ & CEN - MAT $\alpha 1$ MAT $\alpha 2$ & $7.2 \%$ \\
& CEN-MAT $\alpha 1$ mat $\alpha 2 \Delta 188$ & $<0.2 \%$ \\
& $2 \mu-$ MAT $\alpha 1$ MAT $\alpha 2$ & \\
$2 \mu-$ MAT $\alpha 1$ mat $\alpha 2 \Delta 188$ & $8.0 \%$ \\
& & $<0.2 \%$
\end{tabular}
tests) on SD medium, selecting for growth of diploids. Transformants mating with the $\alpha$ strain are of the a mating type. nm indicates that no mating was detected with either tester strain. The low amount of a mating seen in the MATa cells carrying the MATa2 or the mata2 $\Delta 188$ plasmid is likely attributable to phenotypic lag following loss of the plasmid. $(B \mid$ The ability of $\alpha 2 \Delta 188$ to allow sporulation was tested. For a mat $\Delta / M A T$ a diploid carrying the plasmid indicated, 500 cells were counted after 3 days at $30^{\circ} \mathrm{C}$ on sporulation medium. The percentage of cells that contained asci is given.

cells do not produce $\alpha$-factor; both $\alpha 1$ and STE12 are required to activate the $\alpha$-factor structural genes (Fields et al. 1988; Dolan and Fields 1990), and both $M A T \alpha 1$ and STE12 are turned off by the al $/ \alpha 2$ repression activity present in a/ $\alpha$ cells (Klar et al. 1981; Strathern et al. 1981; Fields and Herskowitz 1987). However, when fulllength $\alpha 2$ is replaced by $\alpha 2 \Delta 188$, the resultant cells (Fig. $3 \mathrm{~A}$, line 6) produce $\alpha$-factor, indicating that $\alpha 2 \Delta 188$ and al fail to turn off at least one haploid-specific gene (e.g., STE12 or MAT $\alpha 1$ ). We note that in these cells, $\alpha 2 \Delta 188$ is repressing the a-specific genes, as no a-factor is produced. These results indicate that $\alpha 2 \Delta 188$ is deficient in working with al to turn off the haploid-specific genes but competent in working with MCM1 to turn off the a-specific genes.

Mating tests confirm this conclusion. a/ $\alpha$ cells normally do not mate with either a or $\alpha$ cells because the $\alpha$-specific, a-specific, and haploid-specific genes are all shut off. However, a $/ \alpha$ cells that contain $\alpha 2 \Delta 188$ instead of $\alpha 2$ mate as $\alpha$ cells, indicating that the haploid-specific genes are not turned off. Moreover, the ability of these cells to mate as $\alpha$ cells indicates that the a-specific genes are being repressed by $\alpha 2 /$ MCM1.

Finally, we examined the ability of $\alpha 2 \Delta 188$ to promote sporulation. In response to nutrient starvation, normal a/ $\alpha$ cells undergo meiosis and package the meiotic products into spores. In addition to the appropriate starvation conditions, this process requires that the $R M E 1$ gene be shut off by al and $\alpha 2$ (see Mitchell and Herskowitz 1986). As shown in Figure 3B, an a $\alpha$ cell that contains $\alpha 2 \Delta 188$ instead of $\alpha 2$ fails to undergo sporulation. Although there are many possible explanations for the failure of this strain to sporulate, the simplest is that the $R M E 1$ gene fails to be repressed by al and $\alpha 2 \Delta 188$.

\section{Effects of $\alpha 2$ truncations on the repression of transcription from reporter gene constructs}

From the mating and sporulation observations of Figure 3 we concluded that $\alpha 2 \Delta 188$ can repress a-specific genes but not haploid-specific genes. To test this hypothesis in a more direct way, we checked the ability of $\alpha 2 \Delta 188$ to repress transcription of a reporter gene whose expression is controlled by either an a-specific or a haploid-specific gene operator.

When an a-specific gene operator is placed in a heterologous promoter, the new promoter comes under control of $\alpha 2 / \mathrm{MCMl}$ (Johnson and Herskowitz 1985). Similarly, a1 $/ \alpha 2$ represses a heterologous promoter that contains a haploid-specific gene operator (Miller et al. 1985; Goutte and Johnson 1988). In the experiment of Figure 4 we utilized promoter constructions containing the upstream region of CYC1 fused to an Escherchia coli lacZ reporter gene (Guarente and Hoar 1984). These constructs were integrated in single copy into the yeast hap- 
A

mat $\Delta$

$$
\begin{aligned}
& \text { CEN - vector only } \\
& \text { CEN - MAT } \alpha 1 \text { MAT } \alpha 2 \\
& \text { CEN - MAT } \alpha 1 \text { mat } \alpha 2 \Delta 188
\end{aligned}
$$

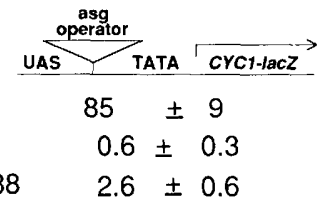

B

MATa

CEN - vector only

CEN - MAT $\alpha 1 M A T \alpha 2$

CEN - MAT $\alpha 1$ mat $\alpha 2 \Delta 188$

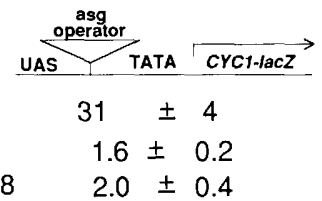

C

MATa

$\overline{\text { MAT } \alpha 1 \text { MAT } \alpha 2}$

$\begin{array}{ll}2 \mu \text { - vector only } & 1.1 \pm 0.2 \\ 2 \mu-\text { MAT } \alpha 1 \text { MAT } \alpha 2 & 0.5 \pm 0.1 \\ 2 \mu-\text { MAT } \alpha 1 \text { mat } \alpha 2 \Delta 188 & 2.7 \pm 0.3\end{array}$

mat $\triangle$

MAT $\alpha 1$ MAT $\alpha 2$

$\begin{array}{lcc}2 \mu \text {-vector only } & 40 & \pm 4 \\ 2 \mu \text {-MAT } \alpha 1 \text { MAT } \alpha 2 & 26 & \pm 3 \\ 2 \mu \text {-MAT } \alpha 1 \text { mat } \alpha 2 \Delta 188 & 28 & \pm 3\end{array}$

\begin{tabular}{c} 
UAS, TATA $\stackrel{\overrightarrow{c Y c \text { l-lacz }}}{\longrightarrow}$ \\
\hline $59 \pm 5$ \\
$63 \pm 6$ \\
$63 \pm 8$
\end{tabular}

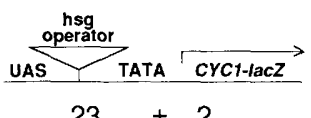

$23 \pm 2$

$1.4 \pm 0.2$

$22 \pm 4$

Figure 4. (A) mat $22 \Delta 188$ represses a test promoter that contains an a-specific gene operator. For each experiment, the reporter construct, indicated across the top, was integrated at the URA3 locus of a mat $\Delta$ strain. Each reported value is the average of three $\beta$-galactosidase assays performed on three transformants $(n=9)$. The a-specific gene operator is that found upstream of the STE6 gene. (B) mata2 2188 fails to repress a test promoter that contains a haploid-specific gene operator. The basic plan of the experiment is described above in $A$. In this case, the reporter constructs were integrated into the genome of a $M A T$ a strain, which contains al protein. In addition, a reporter containing a haploid-specific gene (hsg) operator, that from upstream of the MAT $\alpha 1$ gene (Astell et al. 1981; Siliciano and Tatchell 1984, 1986), was utilized. $(C)$ mata $2 \Delta 188$ has a slight dominant negative effect. Each diploid has a reporter at both URA3 loci and carries the indicated plasmids.

loid genome. Where indicated in Figure 4, an operator was present between the upstream activating sequence (UAS) and TATA regions of the promoter. Promoter activity was assayed by measuring the level of $\beta$-galactosidase activity.

As shown in Figure 4, whereas a reporter bearing an a-specific gene operator is efficiently repressed by $\alpha 2 \Delta 188$ (Fig. 4A, lines 1,3), a reporter carrying a haploidspecific gene operator reporter is not (Fig. 4B, lines 1,3 ). These results are consistent with those of the halo, mating, and sporulation tests. We also determined whether the $\alpha 2 \Delta 188$ mutation is dominant, that is, whether expression of $\alpha 2 \Delta 188$ interferes with the ability of fulllength $\alpha 2$ to repress via a haploid-specific gene operator. When overproduced on a high-copy plasmid, $\alpha 2 \Delta 188$ has only a very slight dominant effect on al $/ \alpha 2$ repression, indicating that $\alpha 2 \Delta 188$ does not inactivate the al protein in a cell (Fig. 4C).

The repression defect of $\alpha 2 \Delta 188$ is attributable to a defect in binding the haploid-specific gene operator with a1

One hypothesis for the failure of $\alpha 2 \Delta 188$ to repress via a haploid-specific gene operator is that $\alpha 2 \Delta 188$ and al fail to occupy the operator in vivo as a result of a DNAbinding deficiency. According to this idea, $\alpha 2 \Delta 188$ would retain the ability to efficiently bind with MCM1 to the a-specific gene operator. To test this hypothesis, $\alpha 2$ and $\alpha 2 \Delta 188$ were expressed in E. coli and purified to apparent homogeneity (see Materials and methods). We compared the abilities of the proteins to bind DNA by using a mobility shift assay in which free DNA is separated from protein-bound DNA by gel electrophoresis (Fried and Crothers 1981).

Although at the concentrations used in this experiment neither al nor $\alpha 2$ alone binds the haploid-specific gene operator, together the proteins bind cooperatively to form a complex consisting of one molecule of each al, $\alpha 2$, and operator (Dranginis 1990; Goutte and Johnson 1993). Figure 5A shows the results of a mobility shift experiment in which a radioactively labeled DNA fragment that contains a haploid-specific gene operator is bound by al in the presence of a constant amount of purified $\alpha 2$ (left) or $\alpha 2 \Delta 188$ (right). The reactions contained consecutive twofold dilutions of purified al, beginning with lanes a. From this experiment we conclude that $\alpha 2 \Delta 188$ requires $>100$-fold more a1 to occupy $50 \%$ of the haploid-specific gene operators than does the full-length $\alpha 2$.

Although defective in binding with al, $\alpha 2 \Delta 188$ retains the ability to bind cooperatively with MCM1. In Figure $5 \mathrm{~B}$, a radioactively labeled DNA fragment that contains an a-specific gene operator is bound by MCM1 (left), by MCM1 together with purified $\alpha 2$ (center), or by MCM1 together with purified $\alpha 2 \Delta 188$ (right). For these experiments, the MCMl was provided in a crude yeast extract. The lanes contain consecutive threefold dilutions of the MCM1-containing extract beginning with lanes $b$. Lanes a contain no extract. We conclude from Figure 5 that $\alpha 2 \Delta 188$ is defective in binding a haploid-specific gene 

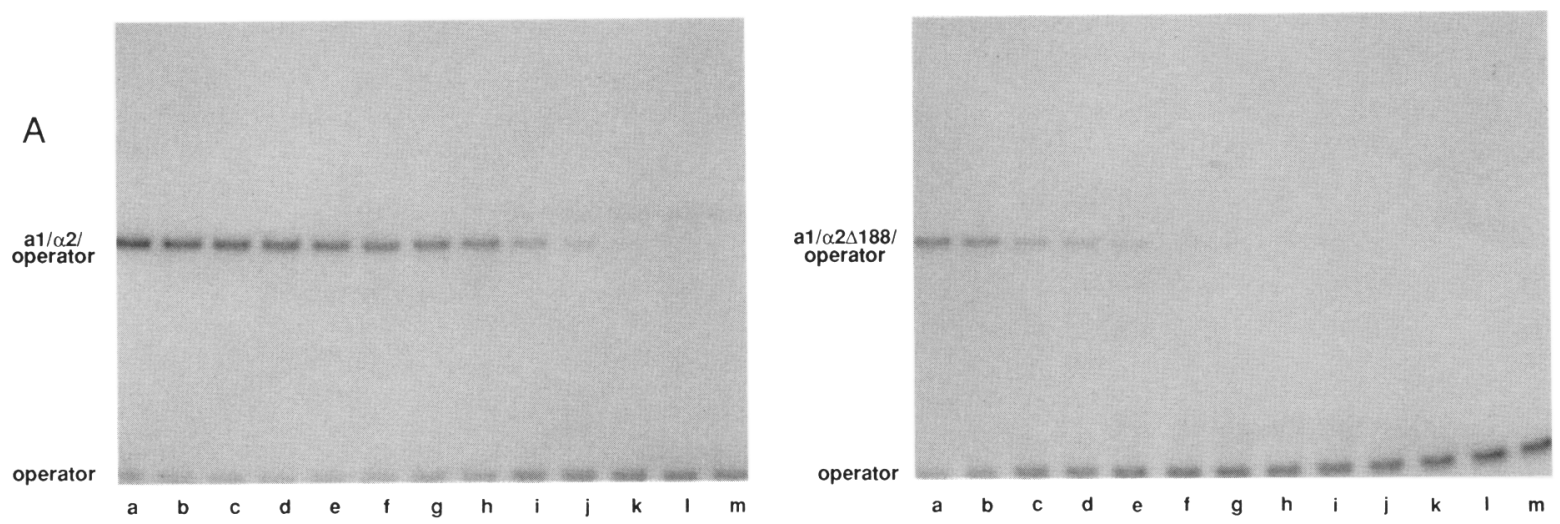

B
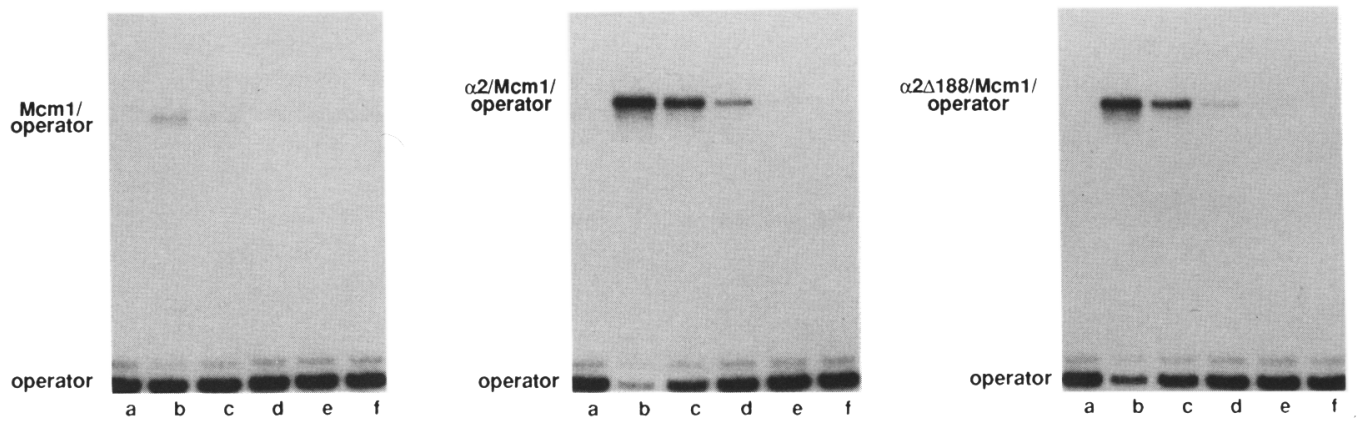

Figure 5. $\alpha 2 \Delta 188$ is defective in binding with al to a haploid-specific gene operator but binds as well as full-length $\alpha 2$ with MCM1 to an a-specific gene operator. $(A)$ (Left) The results of adding decreasing amounts of al protein to a constant concentration of full-length $\alpha 2\left(10^{-7} \mathrm{M}\right)$. A ${ }^{32} \mathrm{P}$-labeled DNA fragment containing a haploid-specific operator [the consensus site described in Goutte and Johnson (1993)] was also included, and protein-DNA complexes were visualized as the decreased mobility of the labeled DNA fragment. Lanes $a-m$ contain successive two-fold dilutions of al protein beginning with a concentration of $1.4 \times 10^{-6} \mathrm{M}$ in lane $a$. (Right) The results of the same experiment performed with $\alpha 2 \Delta 188$ instead of full-length $\alpha 2$. (B) (Left) The binding of MCM1 (in an extract prepared from mat $\Delta$ cells) to a DNA fragment containing an a-specific gene operator (that from upstream of STE6). Lane $a$ contains no extract; lanes $b-f$ contain successive threefold dilutions of the extract beginning with lane $b$. The titration was repeated in the presence of $2.5 \times 10^{-8} \mathrm{M}$ full-length $\alpha 2$ (center) and in the presence of $2.5 \times 10^{-8} \mathrm{M} \alpha 2 \Delta 188$ (right).

operator with al but competent to bind an a-specific gene operator cooperatively with MCM1.

\section{$a 1 / \alpha 2 \Delta 188$ protects the same regions of operator as does $\mathrm{a} 1 / \alpha 2$}

By use of high concentrations of al and $\alpha 2 \Delta 188$ we were able to compare the protection patterns produced by $\mathrm{a} 1 /$ $\alpha 2 \Delta 188$ and al $/ \alpha 2$ on a haploid-specific gene operator. al $/ \alpha 2 \Delta 188 /$ operator and a1/ $\alpha 2 /$ operator complexes that were exposed to attack by hydroxyl radicals (not shown) or DNase I (Fig. 6) showed only one difference in their protection patterns. The DNase I protection pattern of the al/ $\alpha 2 \Delta 188$ /operator complex lacks one enhancement (indicated by the arrow) that is seen with the wildtype al/ $\alpha 2$ operator complex. Interference experiments using operators chemically modified by either dimethylsulfate or formic acid give similar patterns for al/ $\alpha 2 \Delta 188$ /operator and al $/ \alpha 2 /$ operator (data not shown). Taken together, the data indicate that the $\alpha 2$ tail does not make an obvious contribution to the protection of operator DNA.

\section{$\alpha 2 \Delta 188$ forms a less stable complex with a1 and operator than does $\alpha 2$}

The reduced amount of a $1 / \alpha 2 \Delta 188 /$ operator complex at equilibrium compared with a1/ $\alpha 2 /$ operator (Fig. 5 ) is attributable to the a1/ $\alpha 2 \Delta 188 /$ operator complex forming more slowly and/or falling apart more rapidly than the a1 $/ \alpha 2 /$ operator complex. In contrast to the rate of association, the rate of complex dissociation can be accurately measured without precise knowledge of the concentrations of active proteins in the reaction. We therefore compared rates of dissociation of the a $1 / \alpha 2 /$ operator and the a1/ $\alpha 2 \Delta 188 /$ operator complexes. al $/ \alpha 2 /$ operator and a1/ $\alpha 2 \Delta 188 /$ operator complexes were allowed to form, and an excess of unlabeled operator was then added to the reaction. At the indicated times after the addition of unlabeled operator, aliquots of the reactions were 

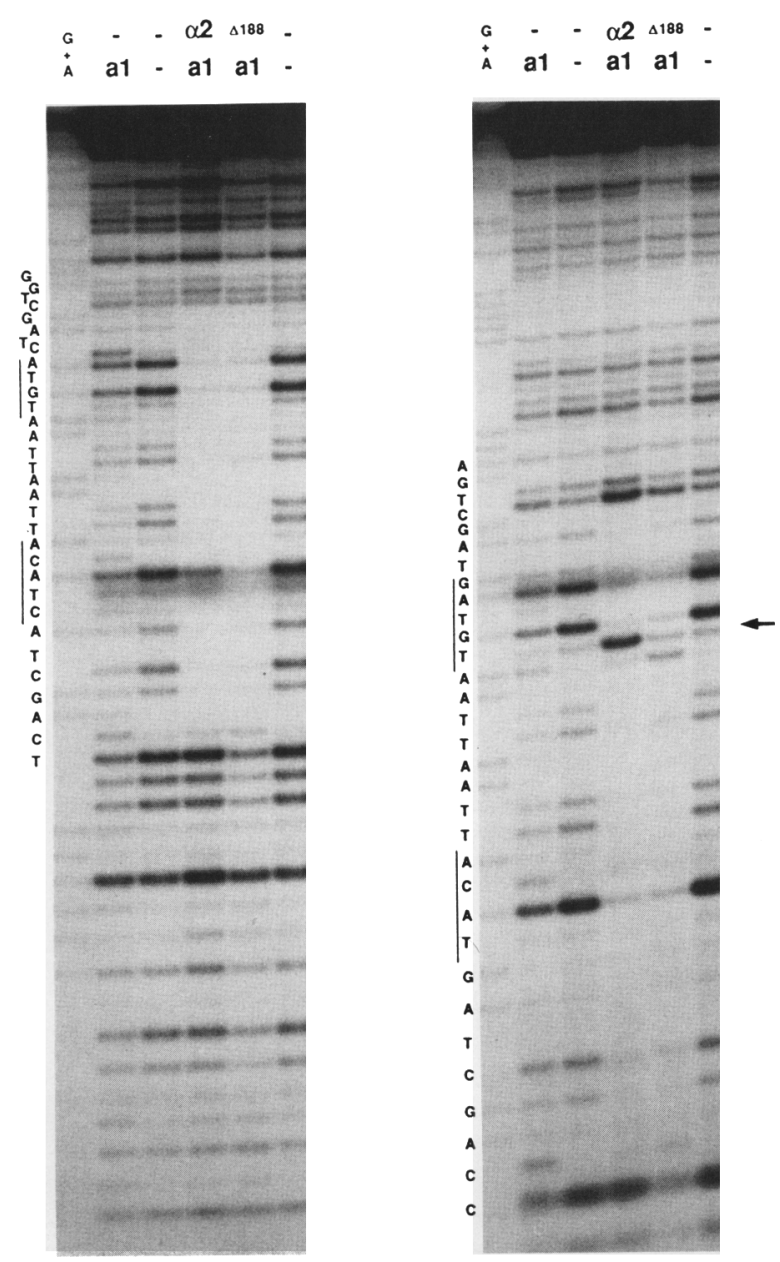

Figure 6. DNase I protection patterns produced by the binding of al $/ \alpha 2$ to the consensus haploid-specific operator. Shown are the results obtained with the bottom strand labeled (left) and the top strand labeled (right). The vertical lines highlight the conserved portions of the consensus hsg operator, whose sequence is given (see Goutte and Johnson 1993). The arrow indicates a site of enhanced DNase I cleavage in the al/ $\alpha 2 /$ operator complex but absent from the al/ $\alpha 2 \Delta 188$ /operator complex.

loaded on a running gel to visualize the amount of remaining complex. Figure 7 shows that the a1/ $\alpha 2 /$ operator complex remains intact much longer than the al/ $\alpha 2 \Delta 188$ /operator complex. The half-life of the a1/ $\alpha 2 /$ operator complex under these conditions is $\sim 16 \mathrm{~min}$, whereas the a1/ $\alpha 2 \Delta 188$ /operator complex is fully dissociated within $0.5 \mathrm{~min}$. The difference between the rates of dissociation of the a1/ $\alpha 2 \Delta 188 /$ operator complex and the a1/ 2 /operator complex is large enough to account for the difference in the equilibrium amounts of the complexes determined in Figure 5. That is, the instability of the complex can account for the observed decrease in DNA binding. This decrease in binding can in turn explain the observed failure of $\alpha 2 \Delta 188$ to repress the transcription of haploid-specific genes in vivo.

\section{Discussion}

$\alpha 2$ represses two sets of genes, the haploid-specific genes and the a-specific genes: $\alpha 2$ and al together recognize haploid-specific gene operators, and $\alpha 2$ and MCM1 together recognize a-specific gene operators. We are interested in the features of $\alpha 2$ that permit it to work in combination with both of these proteins. In this paper we examine the function of the carboxy-terminal tail of the $\alpha 2$ protein. Two observations demonstrate that this tail, which extends from the $\alpha 2$ homeo domain, is structurally disordered. First, we show here that the tail is much more sensitive to protease attack than is the adjacent homeo domain. Second, NMR and X-ray crystallography studies have shown that $\alpha 2_{128-210}$ is ordered through the end of the homeo domain, yet the tail (amino acids 189-210) appears disordered (Phillips et al. 1991; Wolberger et al. 1991). Experiments presented in this paper reveal that the carboxy-terminal tail stabilizes the complex that $\alpha 2$ forms with al and the haploid-specific gene operator. As a consequence, $\alpha 2$ mutants that lack the tail are deficient in repression of the haploidspecific genes. In contrast, the $\alpha 2$ tail is not required for $\alpha 2$ to work with MCM1.
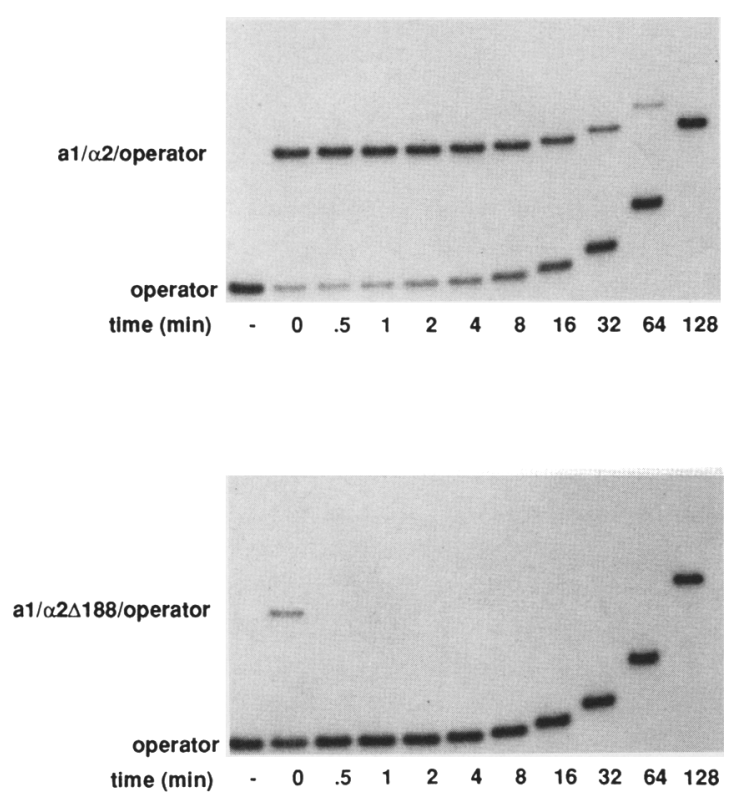

Figure 7. The a1 $/ \alpha 2 \Delta 188$ /operator complex is less stable than the a1/ $\alpha 2$ /operator complex. Shown are the results of off-rate experiments in which the radioactively labeled DNA fragment containing the consensus haploid-specific gene operator was prebound with either $1 \times 10^{-8} \mathrm{M} \alpha 2$ and $5 \times 10^{-8} \mathrm{M}$ al (top, 0 min) or $2 \times 10^{-8} \mathrm{M} \alpha 2 \Delta 188$ and $60 \times 10^{-8} \mathrm{M}$ al (bottom, $0 \mathrm{~min}$ ). Excess unlabeled operator was added to the reactions at time zero, and aliquots were taken at the indicated times and loaded on a running gel to visualize the amount of complex remaining. The lanes marked with a hyphen contain no $\alpha 2, \alpha 2 \Delta 188$, or $a 1$. The half-life of the al/ $\alpha 2 /$ operator is $\sim 16 \mathrm{~min}$ under these conditions and that for the al $/ \alpha 2 \Delta 188 /$ operator is less than $30 \mathrm{sec}$. Because the electrophoresis was continuous during the experiment, the DNA fragments are located progressively higher in the gel with increasing time, because the later samples were electrophoresed for shorter periods of time than were the earlier samples. 
Comparison of the behavior of several carboxy-terminal truncation mutants of $\alpha 2$ (Fig. 1) has allowed us to narrow the region of importance for $\alpha 2$ tail function. The truncation of $\alpha 2$ that ends with residue 203 has wildtype activity, indicating that residues $203-210$ of $\alpha 2$ are dispensable for function. In contrast, a mutant deleted for an additional five residues $(\alpha 2 \Delta 198)$ is, like $\alpha 2 \Delta 188$, incapable of repressing haploid-specific genes while still able to repress a-specific genes. Considered together, these observations indicate that positions 198-203 of $\alpha 2$ contain residues required for function with al.

The inability of tailless $\alpha 2(\alpha 2 \Delta 188)$ to stably bind DNA with al could in principle be attributable to a defect in contact with al, in contact with the DNA, or both. The similarity in DNA protections found in al/ $\alpha 2 \Delta 188$ /operator and al $/ \alpha 2 /$ operator suggests that the $\alpha 2 \Delta 188$ defect does not lie in DNA contact; that is, the tail seems unlikely to interact with a specific patch of DNA. Thus, it seems likely that the defect in $\alpha 2 \Delta 188$ lies in its interaction with al. The idea that the carboxyterminal tail of $\alpha 2$ interacts directly with al is supported by NMR spectroscopy studies of $\alpha 2_{128-210}$ : The addition of al to a solution of $\alpha 2_{128-210}$ (in the absence of DNA) produces a number of resonance changes in the $\alpha 2$ tail but not in the $\alpha 2$ homeo domain (C. Phillips and F. Dahlquist, pers. comm.).

The role of the $\alpha 2$ carboxy-terminal tail, as defined by this study, is consistent with mutants of $\alpha 2$ identified previously in other laboratories. A missense mutation in the tail (at $\mathrm{Leu}_{196}$ ) has the same phenotype in vivo as does the $\alpha 2 \Delta 188$ truncation (Strathern et al. 1988). A mutant of $\alpha 2$ that contains an additional 28 amino acids at the end of $\alpha 2$ also has the same phenotype (Tatchell et al. 1981), possibly because the extra long tail somehow obstructs proper contact with al or facilitates degradation of the tail.

The fact that $\alpha 2 \Delta 188$ and al still show some cooperativity of binding suggests that the tail is not the only region of $\alpha 2$ that interacts with al. Several lines of evidence suggest that additional contacts are provided by the amino-terminal region of $\alpha 2$ : Harashima et al. (1989) have identified missense mutations at position 33 in the amino-terminal region of $\alpha 2$ that are defective in a1/ $\alpha 2$ repression but not $\alpha 2 / \mathrm{MCM} 1$ repression, possibly because of interference with the interaction with al but not MCM1. Deletion studies indicate that residues 2163 of $\alpha 2$ are required for normal binding of DNA with al (Goutte and Johnson 1988). Finally, $\alpha 2_{136-210}$, which lacks the entire amino-terminal domain, is reduced $\sim 10$ fold in binding with al (C. Goutte, M. Stark, and A.D. Johnson, unpubl.). These findings indicate that in addition to the carboxy-terminal tail, an amino-terminal region of $\alpha 2$, which is at least partially contained within residues 33-71, is required for efficient DNA binding and with al and $\alpha 2$. It is possible, for example, that the amino-terminal regions of $\mathbf{a} 1$ and $\alpha 2$ form a heterodimer and that the additional interaction between the $\alpha 2$ tail and al position the two homeo domains with the correct orientation to recognize the haploid-specific operator.

A picture of $\alpha 2$ has emerged in which the selection of target genes is carried out by the homeo domain in conjunction with two flexible extensions, one on each side of the homeo domain. To the amino-terminal side of the homeo domain is an extension that is required for interaction with MCM1 (Vershon and Johnson 1993). To the carboxy-terminal end of the homeo domain is an extension that is now known to be required for function with al. This arrangement-a homeo domain flanked by extensions-might occur in other homeo domain proteins (see Niølstad et al. 1988; Zeng et al. 1993). For example, the zebrafish ZF-21 and the mouse Hox 2.1 homeo domain proteins show a very high degree of amino acid similarity, which includes 46/66 identical residues immediately amino-terminal to the homeo domain and 16/ 16 residues carboxy-terminal to the homeo domain. Thirty-seven of the 66 amino-terminal extension residues and 14 of the 16 tail residues of these proteins are also conserved in the XlHox4 protein (Niølstad et al. 1988). Such conservation of the regions to either side of the homeo domain may be attributable to the requirement for these proteins to interact with similar partner proteins through flexible extensions.

\section{Materials and methods}

Plasmids and strains

To produce $\alpha 2 \Delta 188$, codon 189 was replaced by a stop codon via oligonucleotide-directed mutagenesis (Kunkel et al. 1987) of the a2-coding sequence using the oligonucleotide 5'-GCGATTGTTATCTATTTTTC-3' and pAV99 (Vershon and Johnson 1993) a plasmid containing $\mathrm{P}_{\mathrm{tac}}-\alpha 2$ from pDS1 (Sauer et al. 1988) and a bacteriophage $\mathrm{fl}$ origin of replication. The other truncations shown in Figure 1 were made in the same manner with the following oligonucleotides. $\alpha 2 \Delta 206: 5$-TTCTTTCTTCTATGCC-3'; $\alpha 2 \Delta 203$ : 5'-GCCAGCTACTCACCGCT-3'; $\alpha 2 \Delta 198$ : 5'-GCTCAACTAGTCCGCT-3'. For overexpression in E. coli, the $1.0-\mathrm{kb} B a m H I$ fragment was removed from downstream of the $\alpha 2 \Delta 188$-coding sequence. The resulting plasmid (pAM24) was then transformed using the $\mathrm{CaCl}_{2}$ method into an $E$. coli strain containing an $\mathrm{F}^{\prime}$ lacI $I^{\mathrm{Q} 1}$.

Yeast centromere plasmids used were pAV114 (CEN-vector of Figs. 3 and 4), which is pRS315 (Sikorski and Hieter 1989) in which the BamHI site has been cut, filled, and religated by $A$.

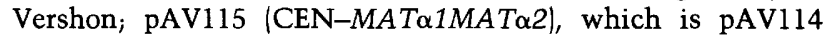
with the 4.3-kb HindIII MATa fragment (Astell et al. 1981) cloned into the HindIII site by A. Vershon; and pAM25 (CEN$M A T \alpha 1 m a t \alpha 2 \Delta 188$ ), which was made by replacing the $0.6-\mathrm{kb}$ BglII-BamHI fragment of MAT 22 in pAV115 with the $0.6-\mathrm{kb}$ BgIII-BamHI fragment of pAM24.

Yeast high-copy plasmids used include YEp13; pKK19 $(2 \mu-$ $M A T \alpha 1 M A T \alpha 2$ of Figs. 3 and 4), which is YEp13 with the $4.3-\mathrm{kb}$ HindIII fragment of MAT $\alpha$ cloned into the HindIII site of YEp13 by $\mathrm{K}$. Komachi; and pAM26 $(2 \mu-M A T \alpha 1$ mat $\alpha 2 \Delta 188)$, which is the 4.3-kb HindIII fragment of pAM25 cloned into the HindIII site of YEpl3.

Yeast plasmids were introduced by lithium acetate transformation as described by Ito et al. (1983) into his4 leu2 trp1 ura3 strains varying only at the MAT locus. a cells are EG123, $\alpha$ cells are 246.1.1, and mat $\Delta$ cells are KT23 $\alpha \times 8$ (Tatchell et al. 1981). $M A T a / m a t \Delta$ diploids were made by mating a mat $\Delta$ strain containing a MATa leu2 plasmid with a MATa strain, selecting for growth of diploids, and then dropping the plasmid by growing 
cells nonselectively and screening for colonies that were unable to grow in the absence of leucine.

Reporter strains were constructed by K. Komachi. Integrating versions of the pLG $\Delta 312 \mathrm{~S}$-derived plasmids described in Keleher et al. (1988) were made, integrated at the URA3 locus, and checked for single-copy integration by DNA-DNA hybridization. The $M A T a / M A T \alpha$ diploid reporter strains were made by mating the appropriate haploids. Strains used for Figure $2 \mathrm{C}$ were checked for overexpression of $\alpha 2 \Delta 188$ and $\alpha 2$ by anti- $\alpha 2$ immunoblots of whole cell lysates.

\section{$\beta$-Galactosidase assays}

$\beta$-galactosidase assays were performed as reported in Smith and Johnson (1992), except all reactions were carried out at $30^{\circ} \mathrm{C}$. Each number reported is the average of three isolates each done in triplicate (i.e., $n=9$ ) \pm the calculated standard deviation of each group.

\section{Halo, mating, and sporulation tests}

Halo and mating tests were performed as described by Sprague (1991). Halo tester strains used were SM 1085 (MATasst2adc) obtained from Susan Michaelis and RC757 (MATa sst2-1 rme1 his6 met1 can1 cyh1) described by Chan and Otte (1982). Mating tests were performed using mating tester strains 227 (MATa cry1 lys1) and 1793 (MATa lys1).

\section{Proteolysis}

Fifteen micromolar $\alpha 2_{128-210}$ was treated with 180 nM endopeptidase Glu-C (preparation of Staphylococcus aureas protease V8 from Boehringer Mannheim) in $50 \mathrm{mM} \mathrm{NH}_{4} \mathrm{HCO}_{3}$ (pH 7.8), 100 $\mathrm{mM} \mathrm{NaCl}$ at $35^{\circ} \mathrm{C}$. Aliquots were removed at the times indicated in the figure, boiled in Laemmli sample buffer, and chilled on ice. Samples were then run on a $19 \%$ acrylamide $/ 0.07 \%$ bis denaturing protein gel followed by staining with Coomassie brilliant blue. Amino-terminal sequencing of the 40-min sample, performed by the Biomolecular Resource Center at UCSF, indicated that the major product began with $T_{128}$.

\section{Protein purification}

$\alpha 2$ was purified as described in Sauer et al. (1988) except that the $\alpha 2$ eluted from the SP-Sephadex column was dialyzed against $U$ buffer and loaded onto a phosphocellulose column, which was then eluted with a linear gradient from $U$ buffer to $U$ buffer $+500 \mathrm{~mm} \mathrm{NaCl}$. Purification then proceeded as described.

$\alpha 2 \Delta 188$ purification was carried out as described for $\alpha 2$ except that fractions from the phosphocellulose column were dialyzed against $\mathrm{S}$ buffer $+500 \mathrm{mM} \mathrm{NaCl}$ and then chromatographed on an LKB Pharmacia Superose 12 HR 30/10 FPLC column.

\section{DNA-binding assays}

Mobility shift assays were performed in $20 \mathrm{~mm}$ Tris (pH 8), 0.1 mM EDTA, $5 \mathrm{~mm} \mathrm{MgCl}_{2}, 2.5 \%$ glycerol, $0.1 \%$ NP-40, $5 \mathrm{~mm}$ DTT, $150 \mathrm{mM} \mathrm{NaCl}, 150 \mu \mathrm{M} \mathrm{BSA}$, and $1 \mu \mathrm{g} / \mathrm{ml}$ of HaeIII-digested E. coli chromosomal DNA. MCMl activity was provided by a mat $\Delta$ cell extract made by $\mathrm{K}$. Komachi as described by Goutte and Johnson (1988). Reactions containing MCM1 activity also contained $20 \mathrm{mM} \mathrm{KCl}$ and $1 \mathrm{~mm} \beta$-mercaptoethanol from the buffer in which they were made. Radioactively labeled 73- or 86-bp DNA fragments containing either the consensus haploid-specific gene operator (Goutte and Johnson 1993) or the STE6 gene operator (Johnson and Herskowitz 1985) were added to the reaction at $10^{-10} \mathrm{M}$. Protein was added to the final con- centrations given in the figure legends. Reactions were mixed and incubated at room temperature for $1 \mathrm{hr}$ before being loaded onto a $6 \% 1 \times$ Tris/borate/EDTA polyacrylamide gel and electrophoresed at $200 \mathrm{~V}$. For the off-rate experiment shown in Figure 5,12 reactions worth of material was set up in one tube and allowed to incubate. One reaction (i.e., one-twelfth) was removed and loaded as a time zero sample onto a gel running at 60 V. Next, unlabeled duplex operator oligonucleotide was added to the remaining reactions. One reaction was removed and loaded onto the running gel at each of the times indicated in Figure 5. After the last time point, the voltage across the gel was increased to $200 \mathrm{~V}$ for $30 \mathrm{~min}$. The test lane is a reaction in which the unlabeled operator was mixed with the proteins before the addition of labeled operator, to test whether the amount of unlabeled operator DNA used was sufficient to compete the proteins off of the labeled operator DNA. For half-life determination, the radioactivity in the gel whose autoradiograph is shown in Figure 7 was quantified by a Molecular Dynamics PhosphorImager and the $\log$ of the fraction of radioactivity (DNA) found in the complex was plotted against time. The dissociation constant was determined (from the fraction bound at the 0 -min time point) to be $1 \times 10^{-16} \mathrm{M}^{2}$ for al/ $22 /$ operator and $3 \times 10^{-14} \mathrm{M}^{2}$ for a $1 / \alpha 2 \Delta 188 /$ operator.

DNase I protection experiments were performed as described in Sauer et al. (1988), except that the buffer contained $0.5 \mathrm{~mm}$ EDTA, $12.5 \mathrm{mM} \mathrm{CaCl}_{2}, 2.25 \mu \mathrm{g} / \mathrm{ml}$ of calf thymus DNA, and 50 $\mathrm{mg} / \mathrm{ml}$ of BSA. Reactions were incubated for $30 \mathrm{~min}$ at $20^{\circ} \mathrm{C}$ before the addition of DNase I.

\section{Acknowledgments}

We thank Kelly Komachi for constructing the reporter strains used in Figure 4, C. Goutte for her gift of purified al protein, and A. Vershon for his gift of $\alpha 2$ expression plasmids. We also thank Kelly Komachi, Brenda Herschbach, Nancy Hollingsworth, and Burk Braun for valuable comments on the manuscript. This work was supported by grants from the National Institutes of Health (GM37049) and the Pew Scholars Program.

The publication costs of this article were defrayed in part by payment of page charges. This article must therefore be hereby marked "advertisement" in accordance with 18 USC section 1734 solely to indicate this fact.

\section{References}

Astell, C.R., L. Ahlstrom-Jonasson, M. Smith, K. Tatchell, K.A. Nasmyth, and B.D. Hall. 1981. The sequence of the DNAs coding for the mating-type loci of Saccharomyces cerevisiae. Cell 27: 15-23.

Brake, A., C. Brenner, R. Najarian, P. Laybourn, and J. Merryweather. 1985. In Protein transport and secretion (ed. M.-J. Gething), pp. 103-108. Cold Spring Harbor Laboratory Press, Cold Spring Harbor, New York.

Chan, R.K. and C.A. Otte. 1982. Isolation and genetic analysis of Saccharomyces cerevisiae mutants supersensitive to GI arrest by a factor and $\alpha$ factor pheromones. Mol. Cell. Biol. 2: $11-20$.

Dolan, J.W. and S. Fields. 1990. Overproduction of the yeast Ste12 protein leads to constitutive transcriptional induction. Genes \& Dev. 4: 492-502.

-1991. Cell-type-specific transcription in yeast. Biochim. Biophys. Acta. 1088: 155-169.

Dranginis, A.M. 1990. Binding of yeast al and $\alpha 2$ as a heterodimer to the operator DNA of a haploid-specific gene. Nature 347: 682-685.

Fields, S. and I. Herskowitz. 1987. Regulation by the yeast mating-type locus of STE12, a gene required for expression of 
two sets of cell-type-specific genes. Cell 42: 923-930.

Fields, S., D.T. Chaleff, and G.F. Sprague. 1988. Yeast STE7, STE11, and STE12 genes are required for expression of celltype-specific genes. Mol. Cell. Biol. 8: 551-556.

Fried, M. and D.M. Crothers. 1981. Equilibria and kinetics of lac repressor-operator interactions by polyacrylamide gel electrophoresis. Nucleic Acids Res. 9: 6505-6525.

Goutte, C. and A.D. Johnson. 1988. al protein alters the DNA binding specificity of $\alpha 2$ repressor. Cell 52: 875-882.

- 1993. The yeast al and $\alpha 2$ homeo domain proteins form a DNA binding activity with properties distinct from those of either protein. J. Mol. Biol. (in press).

Guarente, L. and E. Hoar. 1984. Upstream activation sites of the CYC1 gene of Saccharomyces cerevisiae are active when inverted but not when placed downstream of the "TATA box." Proc. Natl. Acad. Sci. 81: 7860-7864.

Hall, M.N. and A.D. Johnson. 1987. Homeo domain of the yeast repressor $\alpha 2$ is a sequence-specific DNA-binding domain but is not sufficient for repression. Science 237: 1007-1012.

Harashima, S., A.M. Miller, K. Tanaka, K.-I. Kusumoto, K.-I. Tanaka, Y. Mukai, K. Nasmyth, and Y. Oshima. 1989. Mating-type control in Saccharomyces cerevisiae: Isolation and characterization of mutants defective in repression by a1- $\alpha 2$. Mol. Cell. Biol. 9: 4523-4530.

Herskowitz, I. 1989. A regulatory hierarchy for cell specialization in yeast. Nature 342: 749-757.

Ito, H., Y. Kukada, K. Murata, and A. Kimura. 1983. Transformation of intact yeast cells treated with alkali cations. $J$. Bacteriol. 153: 163-168.

Johnson, A.D. 1992. A combinatorial regulatory circuit in budding yeast. In Transcriptional regulation (ed. S.L. McKnight and K.R. Yamamoto), pp. 975-1006. Cold Spring Harbor Laboratory Press, Cold Spring Harbor, New York.

Johnson, A.D. and I. Herskowitz. 1985. A repressor (MAT $\alpha 2$ product) and its operator control expression of a set of cell type specific genes in yeast. Cell 42: 237-247.

Keleher, C.A., C. Goutte, and A.D. Johnson. 1988. The yeast cell-type-specific repressor $\alpha 2$ acts cooperatively with a noncell-type-specific protein. Cell 53: 927-936.

Klar, A., J. Strathern, J. Broach, and J. Hicks. 1981. Regulation of transcription in expressed and unexpressed mating type cassettes of yeast. Nature 289: 239-244.

Kuchler, K., R.E. Sterne, and J. Thorner. 1989. Saccharomyces cerevisiae STE6 gene product: A novel pathway for protein export in eukaryotic cells. EMBO J. 8: 3973-3984.

Kunkel, T.A., J.D. Roberts, and R.A. Zakour. 1987. Rapid and efficient site-specific mutagenesis without phenotypic selection. In Methods in enzymology (ed. R. Wu and L. Grossman), pp. 367-382, Academic Press, New York.

Kurjan, J. and I. Herskowitz. 1982. Structure of a yeast pheromone gene $(M F \alpha)$ : a putative $\alpha$-factor precursor contains four tandem copies of mature $\alpha$-factor. Cell 30: 933-943.

MacKay, V.L., S.K. Welch, M.Y. Insley, T.R. Manney, J. Holly, G.C. Saari, and M.L. Parker. 1988. The Saccharomyces cerevisiae BAR1 gene encodes an exported protein with homology to pepsin. Proc. Natl. Acad. Sci. 85: 55-59.

Mak, A. 1992. "Interaction of the Saccharomyces cerevisiae homeo domain protein $\alpha 2$ with its co-repressors". Ph.D. Thesis, University of California, San Francisco.

Miller, A.M., V.L. MacKay, and K.A. Nasmyth. 1985. Identification and comparison of two sequence elements that confer cell-type specific transcription in yeast. Nature 314: 598603.

Mitchell, A.P. and I. Herskowitz. 1986. Activation of meiosis and sporulation by repression of the RME1 product in yeast. Nature 319: 738-742.
Niølstad, P.R., A. Molven, I. Hordvik, J. Apold, and A. Fjose 1988. Primary structure, developmentally regulated expression and potential duplication of the zebrafish homeobox gene ZF-21. Nucleic Acids Res. 16: 9097-9111.

Phillips, C.L., A.K. Vershon, A.D. Johnson, and F.W. Dahlquist. 1991. Secondary structure of the homeo domain of yeast $\alpha 2$ repressor determined by NMR spectroscopy. Genes \& Dev. 5: 764-772.

Sauer, R.T., D.L. Smith, and A.D. Johnson. 1988. Flexibility of the yeast $\alpha 2$ repressor enables it to occupy the ends of its operator, leaving the center free. Genes \& Dev. 2: 807-816.

Sikorski, R.S. and P. Hieter. 1989. A system of shuttle vectors and yeast host strains designed for efficient manipulation of DNA in Saccharomyces cerevisiae. Genetics 122: 19-27.

Siliciano, P.G. and K. Tatchell. 1984. Transcription and regulatory signals at the mating type locus in yeast. Cell 37: 969978.

1986. Identification of the DNA sequences controling the expression of the MAT $\alpha$ locus of yeast. Proc. Natl. Acad. Sci. 83: 2320-2334.

Singh, A., E.Y. Chen, J.M. Lugovoy, C.N. Chang, R.A. Hitzeman, and P.H. Seeburg, 1983. Saccharomyces cerevisiae contains two discrete genes coding for the $\alpha$-factor pheromone. Nucleic Acids Res. 11: 4049-4063.

Smith, D.L. and A.D. Johnson. 1992. A molecular mechanism for combinatorial control in yeast: $\mathrm{Mcml}$ protein sets the spacing and orientation of the homeo domains of an $\alpha 2$ dimer. Cell 68: 133-142.

Sprague, G.F. Jr. 1990. Combinatorial associations of regulatory proteins and the control of cell type in yeast. Adv. Genet. 27: 33-62.

- 1991. Assay of yeast mating reaction. Methods Enzymol. 194: 77-93.

Strathern, J.N., J. Hicks, and I. Herskowitz. 1981. Control of cell type in yeast by the mating type locus: the $\alpha 1-\alpha 2$ hypothesis. I. Mol. Biol. 147: 357-372.

Strathern, J., B. Shafer, J. Hicks, and C. McGill. 1988. $\alpha / \alpha$-specific repression by MATa2. Genetics 120: 75-81.

Tatchell, K., K.A. Nasmyth, and B.D. Hall. 1981. In vitro mutation analysis of the mating-type locus in yeast. Cell 27: 25-35.

Vershon, A.K. and A.D. Johnson. 1993. A short, disordered protein region mediates interactions between the homeo domain of the yeast $\alpha 2$ protein and the Mcml protein. Cell 72: $1-20$.

Wolberger, C., A.K. Vershon, B. Liu, A.D. Johnson, and C.O. Pabo. 1991. Crystal structure of a MAT $\alpha 2$ homeo domainoperator complex suggests a general model for homeo domain-DNA interactions. Cell 67: 517-528.

Zeng, W., D.J. Andrew, M.A. Mathies, M.A. Horner, and M.P. Scott. 1993. Ectopic expression and function of the Antp and Scr homeotic genes: The $\mathrm{N}$ terminus of the homeo domain is critical to functional specificity. Development 118: 339352. 


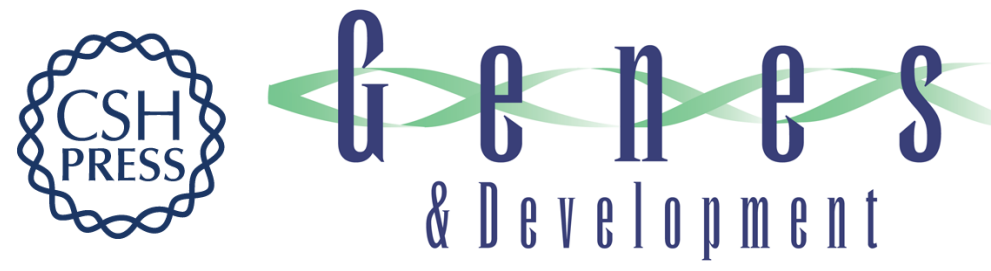

\section{The carboxy-terminal tail of the homeo domain protein alpha 2 is required for function with a second homeo domain protein.}

A Mak and A D Johnson

Genes Dev. 1993, 7:

Access the most recent version at doi:10.1101/gad.7.10.1862

References This article cites 38 articles, 14 of which can be accessed free at:

http://genesdev.cshlp.org/content/7/10/1862.full.html\#ref-list-1

License

Email Alerting

Service

Receive free email alerts when new articles cite this article - sign up in the box at the top right corner of the article or click here.

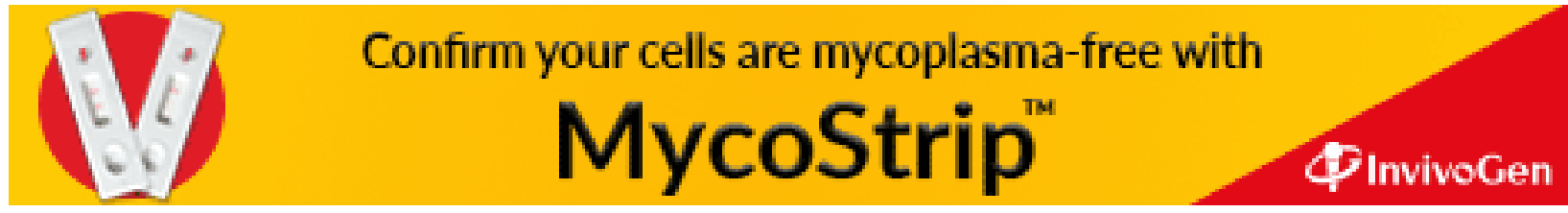

